\title{
Efecto del mate de coca en la función cardio-respiratoria en sujetos jóvenes sometidos a ejercicio sub-máximo, en la exposición aguda a la altura
}

Elydia Mujica, Carlos Saavedra, Julio Huamán, Jesús Díaz, Mario Carrión, Haydée Zúñiga, José Aliaga, José M. Ortiz, Hugo Cebreros, Vidyasagar Casikar, Harold Córdova, Néstor López, Alan Murillo Instituto Nacional de Biologia Andina y Sección Fisiologia, Facultad Medicina, UNMSM

Objetivos: Estudiar el efecto del mate de coca en la función cardio-respiratoria en jóvenes sometidos a ejercicio submáximo, en la exposición aguda a la altura.

Diseño: Experimental.

Institución: Instituto Nacional de Biología Andina y Sección Fisiología, Facultad Medicina, UNMSM.

Participantes: Varones del nivel del mar.

Intervenciones: Se evaluó la función respiratoria y cardiovascular en Lima, a 150 m (NM) y al arribo a Huancayo, a 3280 m (ALT), después de un ejercicio sub máximo y luego de un ejercicio sub máximo después de tomar mate de coca.

Principales medidas de resultados: CVF, VEF1, \%VF1, VEF25-75\%, PEF; PAS, PAD, FC, Sat O2, EKG.

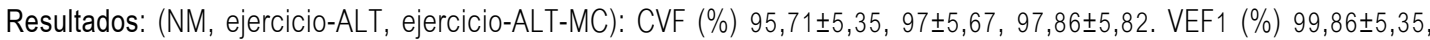

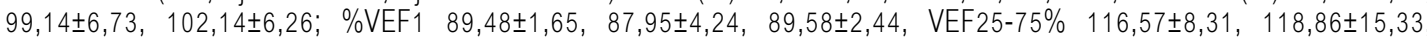

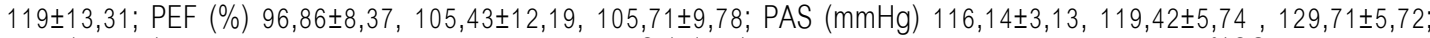
PAD (mmHg) 76,14 $\pm 1,52,77,86 \pm 3,50,76,43 \pm 2,58 ; \mathrm{FC}(\mathrm{p} / \mathrm{min}) 82,71 \pm 2,72,1,66 \pm 1,65,162,14 \pm 1,96 ; \% \mathrm{SO} 289,57 \pm 0,99$, $87,43 \pm 0,72,85,86 \pm 0,83$; ECG sin variaciones.

Conclusiones: Hubo discreto incremento de los valores de función pulmonar luego del ejercicio, sin ser significativos y sin mayores cambios cuando se realizó el ejercicio previa ingesta de una infusión de coca. El EKG no tuvo cambios significativos con respecto a la morfología de sus ondas o complejos entre la basal y aquellos post esfuerzo con y sin mate de coca.

Palabras clave: Mate de coca, función cardio-respiratoria, altura.

\section{Efecto del mate de coca en la función hormonal en sujetos jóvenes sometidos a ejercicio sub-máximo, en la exposición aguda a la altura}

Elydia Mujica, Carlos Saavedra, Julio Huamán, Jesús Díaz, Gerardo Ronceros, Haydée Zúñiga, José Aliaga, Vidyasagar Casikar, Hugo Cebreros, José M. Ortiz, Elizabeth Carranza,

Edgar Florentino

Instituto Nacional de Biología Andina, Instituto Investigaciones Clínicas y Sección Fisiología, Facultad de Medicina, UNMSM

Objetivos: Estudiar el efecto del mate de coca en los niveles hormonales: GH, PRL, ACTH, cortisol, FSH, LH, T, E2.

Diseño: Estudio experimental.

Institución: Instituto Nacional de Biología Andina, Instituto Investigaciones Clínicas y Sección Fisiología, Facultad de Medicina, UNMSM.

Participantes: Varones jóvenes nativos del nivel del mar.

Intervenciones: Se evaluó los parámetros metabólicos en estudio en Lima, 150 m (NM), al arribo a Huancayo, 3280 m $(\mathrm{ALT})$, después de un ejercicio submáximo y luego de un ejercicio submáximo después de tomar mate de coca. Los resultados fueron analizados con el paquete estadístico SPSS 19, mediante la pruebas no paramétricas de Friedman y la prueba de Wilcoxon.

Principales medidas de resultados: GH, PRL, ACTH, cortisol, FSH, LH, T, E2 en suero.

Resultados: (NM, ejercicio-ALT, ejercicio-ALT-MC): GH (mg/dL) 9,82 $\pm 1,03,11,11 \pm 2,42,9,10 \pm 1,03 ; \mathrm{PRL}(\mathrm{mg} / \mathrm{dL})$ $3,94 \pm 0,48,9,81 \pm 1,47(<0,05), 5,51 \pm 1,58(<0,05)$; ACTH (mg/dL) 14,54 $\pm 5,70,10,04 \pm 3,04,15,37 \pm 3,93$; cortisol (mg/ dL) $10,28 \pm 2,18,11,69 \pm 1,08,9,49 \pm 0,64 ; F S H(\mathrm{mg} / \mathrm{dL}) 7,23 \pm 1,03,5,73 \pm 1,54,4,09 \pm 0,76(<0,05) ; \mathrm{LH}(\mathrm{mg} / \mathrm{dL}) 3,74 \pm 1,04$ $3,46 \pm 0,84,1,75 \pm 0,29(<0,05) ; \mathrm{T}(\mathrm{mg} / \mathrm{dL}) 5,27 \pm 0,56,3,27 \pm 0,54(<0,05), 3,42 \pm 0,47(<0,05) ; \mathrm{E} 2(\mathrm{mg} / \mathrm{dL}) 9,39 \pm 1,12$ $11,90 \pm 1,51,9,96 \pm 2,51$.

Conclusiones: Se observó una disminución de PRL y LH después del ejercicio con mate de coca.

Palabras clave: Mate de coca, hormonas, altura. 